\title{
Non-Cooperative Solutions for Claims Problems
}

Citation for published version (APA):

Atlamaz, M., Berden, C., Peters, H. J. M., \& Vermeulen, A. J. (2008). Non-Cooperative Solutions for Claims Problems. Kwantitatieve Economie. METEOR Research Memorandum No. 038 https://doi.org/10.26481/umamet.2008038

Document status and date:

Published: 01/01/2008

DOI:

10.26481/umamet.2008038

Document Version:

Publisher's PDF, also known as Version of record

\section{Please check the document version of this publication:}

- A submitted manuscript is the version of the article upon submission and before peer-review. There can be important differences between the submitted version and the official published version of record.

People interested in the research are advised to contact the author for the final version of the publication, or visit the DOI to the publisher's website.

- The final author version and the galley proof are versions of the publication after peer review.

- The final published version features the final layout of the paper including the volume, issue and page numbers.

Link to publication

\footnotetext{
General rights rights.

- You may freely distribute the URL identifying the publication in the public portal. please follow below link for the End User Agreement:

www.umlib.nl/taverne-license

Take down policy

If you believe that this document breaches copyright please contact us at:

repository@maastrichtuniversity.nl

providing details and we will investigate your claim.
}

Copyright and moral rights for the publications made accessible in the public portal are retained by the authors and/or other copyright owners and it is a condition of accessing publications that users recognise and abide by the legal requirements associated with these

- Users may download and print one copy of any publication from the public portal for the purpose of private study or research.

- You may not further distribute the material or use it for any profit-making activity or commercial gain

If the publication is distributed under the terms of Article $25 \mathrm{fa}$ of the Dutch Copyright Act, indicated by the "Taverne" license above, 
Murat Atlamaz, Caroline Berden, Hans Peters, Dries Vermeulen

Non-cooperative solutions for claims problems

$\mathrm{RM} / 08 / 038$

JEL code: C72

\section{METEबR}

Maastricht research school of Economics of TEchnology and ORganizations

Universiteit Maastricht

Faculty of Economics and Business Administration P.O. Box 616

NL - 6200 MD Maastricht

phone : :+31433883830

fax : ++31433884873 


\title{
Non-cooperative solutions for claims problems*
}

\author{
Murat Atlamaz $\quad$ Caroline Berden ${ }^{\ddagger} \quad$ Hans Peters $^{\ddagger}$ \\ Dries Vermeulen ${ }^{\ddagger}$ \\ This version, October 2008
}

\begin{abstract}
In a claims problem an estate has to be divided among several claimants whose total claim exceeds the size of the estate. This paper extends the noncooperative approach, initiated by O'Neill (1982), by allowing players to put multiple claims on the same part of the estate, and by considering the case where individual claims may exceed the estate. A full characterization of the set of Nash equilibria is obtained both for restricted claims problems, where individual claims do not exceed the estate, and for the general case. Variations on the claim game are considered, which result in proportional division in equilibrium.
\end{abstract}

\section{Introduction}

In a claims problem - also called bankruptcy problem - players have claims on an estate, and the sum of these claims exceeds the size of the estate. The question is how to distribute the estate among the players, based on their claims. There is a substantial literature on this problem, starting with O'Neill (1982) and Aumann and Maschler (1985). Most of this literature considers claims problems from a cooperative, axiomatic point of view. See Thomson (2003) for a relatively recent overview.

In the present paper we take a non-cooperative approach to the claims problem. This is not entirely new: in fact, our model extends a non-cooperative game already proposed by O'Neill (1982). In our basic model, we think of the estate $E$ as represented by the interval $[0, E]$, on which players can put claims. Each player can partition the interval $[0, E]$ into finitely many subintervals and on each of those subintervals put an integer $(0,1,2, \ldots)$ number of claims. Each

\footnotetext{
* This paper combines independent work of the first author (Atlamaz, 2004) and the last three authors (Berden et al. 2008; also reported in Berden, 2008).

${ }^{\dagger}$ Bates White LLC, 1300 Eye Street NW, Suite: 600, Washington, DC 22030, USA. E-mail address: : murat.atlamaz@bateswhite.com.

${ }^{\ddagger}$ Department of Quantitative Economics, University of Maastricht, P.O. Box 616, 6200 MD Maastricht, The Netherlands. Tel.: +31 43 3883835. Email-addresses: c.berden@ke.unimaas.nl, h.peters@ke.unimaas.nl, d.vermeulen@ke.unimaas.nl.
} 
claim has 'height' 1 , and the total area occupied by player $i$ 's claims should be equal to $c_{i}$, where $c_{i}$ is player $i$ 's total claim as given by the claims problem. The difference with the model proposed by O'Neill (1982) is that we allow players to put more than one claim on one and the same subinterval of $E$. We will actually see that this admits Nash equilibria in the claim game that are excluded by O'Neill's game. Moreover, if a player's total claim $c_{i}$ exceeds the estate, then we cannot but allow for multiple claims.

Another interpretation of our claim game is to think of the interval $[0, E]$ as a continuum of uniformly distributed consumers (cf. Hotelling, 1929), and of the claimants as firms who provide services to these consumers, with firm $i$ providing total service $c_{i}$. In this case, each part of the consumer continuum is distributed proportionally with respect to the claims - now called investments - of the firms. Note that this interpretation naturally allows for competitive investments by different firms in one and the same consumer segment, thus, for multiple claims in the bankruptcy terminology.

In still other settings the proportions induced by the claims of the players may be interpreted as probabilities of winning in, for instance, political elections (cf. Merolla, Munger, and Tofias, 2003) or auctions (cf. Cramton, Gibbons, and Klemperer, 1987). There is also a connection with the classical Colonel Blotto game (Borel, 1921). In the present paper, however, we will stick to the bankruptcy problem interpretation.

We first treat restricted claims problems, in which claims do not exceed the estate $\left(c_{i} \leq E\right.$ for each $\left.i\right)$. We characterize all Nash equilibria of our game, and the associated payoff distributions. This case was also studied by O'Neill (1982). As already mentioned, the possibility of multiple claims of a player on one and the same part of the estate allows for additional Nash equilibria. Roughly, these equilibria are advantageous for larger claimants (see Example 4.8).

Restricted claims problems are, of course, a special case of general (unrestricted) problems. Nevertheless, we study the general case after the restricted case since, for the latter, a more explicit description of Nash equilibria is feasible. We also present a complete characterization for the general (unrestricted) case. In that case, however, Nash equilibria may fail to exist. Further, we show that Nash equilibrium payoffs converge to proportional distribution of the estate with respect to the claims as these claims become large.

In our basic model we assume that a player can claim some interval a nonnegative integer number of times. In the paper we also investigate what happens if we allow smaller claim heights (e.g., $\frac{1}{2}, \frac{1}{3}, \ldots$ ) and thus 'finer' claims. Not surprisingly, Nash equilibrium distributions converge again to the proportional distribution as the claim height becomes smaller. We may also drop the assumption that players split their claims into finitely many parts: as an extreme case, we assume that a strategy of a player is a (continuous) nonnegative function on the estate interval $[0, E]$ with total integral equal to $c_{i}$. Intuitively this should again lead to proportional distribution, and we will show that this is indeed the case, even under more restrictive strategies.

Besides O'Neill (1982) there are other articles which deal with strategic models imposed on claims problems. Chun (1989) defines a game where players pro- 
pose division rules. The non-cooperative solution of this game of rules converges to the outcome associated with the constrained equal awards rule. Dagan et al. (1997) construct a consistency based noncooperative model that supports monotonic consistent cooperative solution concepts. Corchón and Herrero (2004) define a game where players propose awards vectors bounded by claims. GarcíaJurado et al. (2006) define an elementary game which has a unique payoff vector in Nash equilibrium associated with any acceptable bankruptcy rule. Our paper is based on the work of the last three authors (Berden et al. 2008, also reported in Berden, 2008), and the independent work of Atlamaz (2004).

The organization of the paper is as follows. Section 2 explains the basic model. Section 3 derives general necessary conditions for Nash equilibria. In Section 4 we consider restricted claims problems and in Section 5 general problems. Variations on our basic model are discussed in Section 6 , and Section 7 concludes.

\section{The model}

The set of players is $N=\{1, \ldots, n\}$, where $n \geq 2$. A claims problem is a pair $(E, c)$, where $E \in \mathbb{R}$ with $E>0$ is the estate and $c=\left(c_{1}, \ldots, c_{n}\right) \in \mathbb{R}^{N}$ with $c_{i}>0$ for each $i \in N$ and $\sum_{i \in N} c_{i} \geq E$ is the vector of claims.

A payoff vector for $(E, c)$ is a vector $x=\left(x_{1}, \ldots, x_{n}\right) \in \mathbb{R}^{N}$ with $\sum_{i \in N} x_{i} \leq$ $E$. In this paper we assume that payoff vectors are reached as equilibrium outcomes of a non-cooperative game in strategic form. Specifically, for the larger part of the paper, we assume that a strategy of player $i$ consists of a finite partition of the interval $[0, E]$ into subintervals and on each subinterval a nonnegative integer number of (sub-)claims, such that the total amount claimed is equal to $c_{i}$. It is without loss of generality to assume that the strategies of all players have the same partition in common, since otherwise we can always consider the common refinement of the player partitions instead. Thus, we obtain the following definition.

Definition 2.1 A claims profile for the claims problem $(E, c)$ is a triple $(y, \beta, m)$, where

(i) $m \in \mathbb{N}, m \geq 1$.

(ii) $y=\left(y_{0}, \ldots, y_{m}\right) \in \mathbb{R}^{m+1}$ with $0=y_{0}<y_{1}<\ldots<y_{m-1}<y_{m}=E$.

(iii) $\beta=\left(\beta_{1}, \ldots, \beta_{n}\right)$, with $\beta_{i}:\{1, \ldots, m\} \rightarrow\{0\} \cup \mathbb{N}$ such that

$$
\sum_{t=1}^{m} \beta_{i}(t)\left(y_{t}-y_{t-1}\right)=c_{i}
$$

for all $i \in N$.

We assume that for a claims profile $(y, \beta, m)$, each interval $\left(y_{t-1}, y_{t}\right)$ is distributed among the players proportionally with respect to their claims. More 
precisely, let $M=\{1, \ldots, m\}$ and for each $t \in M$ let $\beta_{N}(t)=\sum_{i \in N} \beta_{i}(t)$. For each $i \in N$ and $t \in M$ let

$$
\hat{\beta}_{i}(t)=\left\{\begin{array}{cc}
\frac{\beta_{i}(t)}{\beta_{N}(t)} & \text { if } \beta_{i}(t)>0 \\
0 & \text { if } \beta_{i}(t)=0 .
\end{array}\right.
$$

Then player $i$ receives payoff ${ }^{1}$

$$
x_{i}=\sum_{t=1}^{m} \hat{\beta}_{i}(t)\left[y_{t}-y_{t-1}\right] .
$$

A claims profile is a Nash equilibrium if no player can increase his payoff by reshuffling his claims. Intuitively, this means that the marginal utilities on the intervals in the claims profile should be 'more or less' equal: complete equality is generally not possible since the claims are integers. For simplicity, we will just define Nash equilibrium claims profiles in terms of marginal utilities or, more specifically, marginal gains and losses. ${ }^{2}$

Definition 2.2 A claims profile $(y, \beta, m)$ for claims problem $(E, c)$ is a Nash equilibrium profile (NEP) if $\beta_{N}(t) \geq 1$ for all $t \in M$ and if, moreover, the following condition is satisfied: for all $t, t^{\prime} \in M, t \neq t^{\prime}$, and all $i \in N$, if $\beta_{i}(t) \geq 1$, and $\beta_{N}(t) \geq 2$, then

$$
\frac{\beta_{i}(t)}{\beta_{N}(t)}-\frac{\beta_{i}(t)-1}{\beta_{N}(t)-1} \geq \frac{\beta_{i}\left(t^{\prime}\right)+1}{\beta_{N}\left(t^{\prime}\right)+1}-\frac{\beta_{i}\left(t^{\prime}\right)}{\beta_{N}\left(t^{\prime}\right)} .
$$

Inequality (2) says that the loss incurred by taking away a claim (or part of a claim) from some interval $t$ should be at least as big as the gain obtained by putting that claim on some other interval $t^{\prime}$. A particular consequence of this inequality is that, if $\beta_{i}(t)=\beta_{N}(t)$ for some $i \in N$ and $t \in M$, then $\beta_{i}(t)=\beta_{N}(t)=1$. This follows since there is always a $t^{\prime}$ with $\beta_{i}\left(t^{\prime}\right)<\beta_{N}\left(t^{\prime}\right)$. Thus, if a player is the only one to claim a certain part of the estate, then he claims this part only once.

A restricted claims problem is a claims problem $(E, c)$ with $c_{i} \leq E$ for all $i \in N$. O'Neill (1982) considered restricted claims problems and a noncooperative claim game in which each part can be claimed at most once by the same player. We will see that allowing players to claim more than once leads to a larger set of NEP and NEP payoff vectors. For general claim problems, of course, we cannot avoid allowing multiple claims anyway.

\footnotetext{
${ }^{1}$ Observe that, if each part of the estate is claimed at least once, then these payoffs sum to $E$. We allow that some parts are not claimed, in which case the total payoff is smaller than $E$. In a Nash equilibrium, however, this will not occur.

${ }^{2}$ The following definition can be derived as a result from a more basic definition of Nash equilibrium. See Berden et al. (2008) or Berden (2008) for a detailed proof. The crucial insight is that the marginal losses at the left hand side of (2) increase as we remove more claims from $t$, while the marginal gains at the right hand side decrease as we add more claims to $t^{\prime}$. This indicates, indeed, that condition (2) is not only necessary but also sufficient for Nash equilibrium.
} 


\section{$3 \quad$ Some general results}

Throughout this section $(y, \beta, m)$ is a Nash equilibrium claims profile for the claims problem $(E, c)$. We derive three results, which will be used for later analysis and also have independent interest.

The first lemma says that if some player claims some part $t$ at least twice, then he should put positive claims on each part $t^{\prime}$ of the estate on which total claims do not exceed total claims on $t$.

Lemma 3.1 Let $i \in N, t, t^{\prime} \in M, \beta_{i}(t) \geq 2$, and $\beta_{N}\left(t^{\prime}\right) \leq \beta_{N}(t)$. Then $\beta_{i}\left(t^{\prime}\right) \geq 1$.

Proof. Suppose, contrary to what we wish to prove, that $\beta_{i}\left(t^{\prime}\right)=0$. Then

$$
\begin{aligned}
& \frac{\beta_{i}\left(t^{\prime}\right)+1}{\beta_{N}\left(t^{\prime}\right)+1}-\frac{\beta_{i}\left(t^{\prime}\right)}{\beta_{N}\left(t^{\prime}\right)}=\frac{1}{\beta_{N}\left(t^{\prime}\right)+1} \geq \frac{1}{\beta_{N}(t)+1} \\
& >\frac{2}{\beta_{N}(t)}-\frac{1}{\beta_{N}(t)-1}=\frac{\beta_{N}(t)-2}{\beta_{N}(t)\left(\beta_{N}(t)-1\right)} \\
& \geq \frac{\beta_{N}(t)-\beta_{i}(t)}{\beta_{N}(t)\left(\beta_{N}(t)-1\right)}=\frac{\beta_{i}(t)}{\beta_{N}(t)}-\frac{\beta_{i}(t)-1}{\beta_{N}(t)-1},
\end{aligned}
$$

where the strict inequality follows from straightforward calculation ${ }^{3}$. This contradicts (2) and concludes the proof.

The next lemma shows that that the difference between the numbers of claims on each part of the estate can be at most 2 .

Lemma 3.2 For all $t, t^{\prime} \in M,\left|\beta_{N}(t)-\beta_{N}\left(t^{\prime}\right)\right| \leq 2$.

Proof. Suppose, contrary to what we wish to prove, that there are $t, t^{\prime} \in M$ with $\beta_{N}(t) \geq \beta_{N}\left(t^{\prime}\right)+3$. derive

(i) First, suppose that $\beta_{i}(t) \geq \beta_{i}\left(t^{\prime}\right)+1$ for some $i \in N$. From (2) we easily

$$
\beta_{i}\left(t^{\prime}\right) \geq \beta_{N}\left(t^{\prime}\right)-\left(\beta_{N}(t)-\beta_{i}(t)\right) \frac{\beta_{N}\left(t^{\prime}\right)\left(\beta_{N}\left(t^{\prime}\right)+1\right)}{\beta_{N}(t)\left(\beta_{N}(t)-1\right)},
$$

hence

$$
\beta_{i}\left(t^{\prime}\right) \geq \beta_{N}\left(t^{\prime}\right)-\left(\beta_{N}(t)-\beta_{i}\left(t^{\prime}\right)-1\right) \frac{\beta_{N}\left(t^{\prime}\right)\left(\beta_{N}\left(t^{\prime}\right)+1\right)}{\beta_{N}(t)\left(\beta_{N}(t)-1\right)} .
$$

In turn, this implies

$$
\beta_{i}\left(t^{\prime}\right) \geq \frac{\ell k(k-1)-\ell(\ell+1)(k-1)}{k(k-1)-\ell(\ell+1)}, \quad(* *)
$$

where $k=\beta_{N}(t)$ and $\ell=\beta_{N}\left(t^{\prime}\right)$. Now

$$
\operatorname{RHS}(* *)>\frac{\ell}{2} \Leftrightarrow(k-\ell)(k-\ell-3)+2>0
$$

\footnotetext{
${ }^{3}$ Or from strict convexity of the function $x \rightarrow 1 / x$.
} 
and the RHS of this equivalence is clearly true by our assumptions. Hence, $\beta_{i}\left(t^{\prime}\right)>\ell / 2=\beta_{N}\left(t^{\prime}\right) / 2$. But this means that there can be at most one player $i$ for which $\beta_{i}(t) \geq \beta_{i}\left(t^{\prime}\right)+1$. Thus, all players except possibly one player claim in $t$ at most what they claim in $t^{\prime}$.

(ii) From part (i) it follows that there is a player $i \in N$ with $\beta_{i}(t)-\beta_{i}\left(t^{\prime}\right) \geq$ $\beta_{N}(t)-\beta_{N}\left(t^{\prime}\right)$. From $(*)$ we now have

$$
\beta_{i}\left(t^{\prime}\right) \geq \ell-\left(k-\left(\beta_{i}\left(t^{\prime}\right)+k-\ell\right)\right) \frac{\ell(\ell+1)}{k(k-1)},
$$

which after rearranging terms implies $\beta_{i}\left(t^{\prime}\right) \geq \ell=\beta_{N}\left(t^{\prime}\right)$, hence $\beta_{i}\left(t^{\prime}\right)=\beta_{N}\left(t^{\prime}\right)$, and thus $\beta_{i}(t)=\beta_{N}(t) \geq 3$. This is a contradiction since $(y, \beta, m)$ is an NEP.

We will see later (Section 5) that an NEP with the maximal difference of 2 as in Lemma 3.2 can occur. For restricted claims problems, however, this cannot happen, as we will see in the next section.

The final lemma in this section says that, if a player has a claim on an interval $t$, then he should also have a claim on any interval $t^{\prime}$ on which the total number of claims is at least two less than on $t$.

Lemma 3.3 Let $t, t^{\prime} \in M, i \in N, \beta_{i}(t) \geq 1$, and $\beta_{N}\left(t^{\prime}\right) \leq \beta_{N}(t)-2$. Then $\beta_{i}\left(t^{\prime}\right) \geq 1$.

Proof. Suppose, contrary to what we wish to prove, that $\beta_{i}\left(t^{\prime}\right)=0$. Then

$$
\begin{aligned}
\frac{\beta_{i}\left(t^{\prime}\right)+1}{\beta_{N}\left(t^{\prime}\right)+1}-\frac{\beta_{i}\left(t^{\prime}\right)}{\beta_{N}\left(t^{\prime}\right)} & =\frac{1}{\beta_{N}\left(t^{\prime}\right)+1}>\frac{\beta_{N}(t)-\beta_{i}(t)}{\beta_{N}(t)\left(\beta_{N}\left(t^{\prime}\right)+1\right)} \\
& >\frac{\beta_{N}(t)-\beta_{i}(t)}{\beta_{N}(t)\left(\beta_{N}(t)+1\right)}=\frac{\beta_{i}(t)}{\beta_{N}(t)}-\frac{\beta_{i}(t)-1}{\beta_{N}(t)-1},
\end{aligned}
$$

contradicting (2).

\section{Restricted claims problems}

We first derive some additional results for an NEP in restricted claims problems, and next use these to characterize all NEP in such problems.

In Lemmas 4.1-4.4, claims profile $(y, \beta, m)$ is an NEP for the restricted claims problem $(E, c)$. We denote $\beta_{\max }=\max _{t \in M} \beta_{N}(t)$ and $\beta_{\min }=\min _{t \in M} \beta_{N}(t)$.

The first lemma says that each player has at most one claim in the maximally claimed intervals.

Lemma $4.1 \beta_{i}(t) \leq 1$ for all $i \in N$ and $t \in M$ with $\beta_{N}(t)=\beta_{\max }$.

Proof. Suppose, contrary to what we wish to prove, that $\beta_{i}(t) \geq 2$ for some $i \in N$ and $t \in M$ with $\beta_{N}(t)=\beta_{\max }$. Since $c_{i} \leq E$, there must be a $t^{\prime} \in M$ with $\beta_{i}\left(t^{\prime}\right)=0$, but this contradicts Lemma 3.1.

The next lemma says that the differences in total numbers of claims between intervals can be at most 1 . 
Lemma $4.2 \beta_{\max }-\beta_{\min } \leq 1$.

Proof. If this were not true then, by Lemma 3.2, there would be $t, t^{\prime} \in M$ with $\beta_{N}(t)-\beta_{N}\left(t^{\prime}\right)=2, \beta_{N}(t)=\beta_{\max }, \beta_{N}\left(t^{\prime}\right)=\beta_{\min }$. By Lemma 4.1 there are exactly $\beta_{N}(t)$ different players with a claim (namely, of 1) on $t$. By Lemma 3.3 all these players have at least one claim on $t^{\prime}$, but this is clearly impossible.

The next lemma implies that each player has at most two claims on each interval.

Lemma $4.3 \beta_{i}(t) \leq 2$ for all $i \in N$ and $t \in M$.

Proof. Let $i \in N$. In view of Lemmas 4.1 and 4.2 it is sufficient to prove $\beta_{i}(t) \leq 2$ for $t \in M$ with $\beta_{N}(t)=\beta_{\max }-1$. Suppose, to the contrary, that $\beta_{i}(t) \geq 3$ for such a $t$. Since $c_{i} \leq E$ and by Lemma 3.1, there is $t^{\prime} \in M$ with $\beta_{i}\left(t^{\prime}\right)=0$ and $\beta_{N}\left(t^{\prime}\right)=\beta_{\max }$. By using (2) we obtain

$$
\frac{\left(\beta_{\max }-1\right)-\beta_{i}(t)}{\left(\beta_{\max }-1\right)\left(\beta_{\max }-2\right)} \geq \frac{1}{\beta_{\max }+1}
$$

hence

$$
\frac{\beta_{\max }-4}{\left(\beta_{\max }-1\right)\left(\beta_{\max }-2\right)} \geq \frac{1}{\beta_{\max }+1} .
$$

It is easy to check that the latter inequality cannot hold.

In the final lemma we observe that if the bound of 2 in the preceding lemma is reached, then there must be an interval with at least five claims on it. Consequently, by Lemma 4.1, the number of players is at least five as well.

Lemma 4.4 Let $i \in N$ and $\beta_{i}(t)=2$ for some $t \in M$ with $\beta_{N}(t)=\beta_{\max }-1$. Then $\beta_{\max } \geq 5$.

Proof. As in the proof of Lemma 4.3, there is a $t^{\prime} \in M$ with $\beta_{i}\left(t^{\prime}\right)=0$ and $\beta_{N}\left(t^{\prime}\right)=\beta_{\max }$. By $(2)$,

$$
\frac{2}{\beta_{\max }-1}-\frac{1}{\beta_{\max }-2} \geq \frac{1}{\beta_{\max }+1}
$$

which implies $\beta_{\max } \geq 5$.

Lemmas 3.1 and 4.1-4.4 provide a number of necessary conditions for a claims profile $(y, \beta, m)$ to be an NEP. By Lemma 4.2 in particular it follows that there is a $K \in \mathbb{N}$ such that on each interval there are $K$ or $K+1$ claims. Let $R \in \mathbb{R}$ be the total part of $E$ that is claimed $K+1$ times. Then $0 \leq R<E$ and we must have

$$
\sum_{i \in N} c_{i}=K(E-R)+(K+1) R=K E+R
$$

so $K$ and $R$ are uniquely determined by $E$ and $\sum_{i \in N} c_{i}$. The main result of this section is the following theorem, which says that the necessary conditions provided by the mentioned lemmas are also sufficient. 
Theorem 4.5 Let $(E, c)$ be a restricted claims problem, and let $K \in \mathbb{N}$ and $R \in \mathbb{R}$ with $0 \leq R<E$ be the unique numbers satisfying $\sum_{i \in N} c_{i}=K E+R$. Then a claims profile $(y, \beta, m)$ for $(E, c)$ is an NEP if and only if the following four conditions are satisfied:

(A1) For all $t \in M, \beta_{N}(t) \in\{K, K+1\}$.

(A2) For all $t \in M$ and $i \in N$, if $\beta_{N}(t)=K+1$ then $\beta_{i}(t) \leq 1$.

(A3) For all $t \in M$ and $i \in N$, if $\beta_{N}(t)=K$ then $\beta_{i}(t) \leq 2$.

(A4) For all $t \in M$ and $i \in N$, if $\beta_{i}(t)=2$ then $\beta_{i}\left(t^{\prime}\right) \geq 1$ for all $t^{\prime}$ with $\beta_{N}\left(t^{\prime}\right)=K$, and $K \geq 4$.

Observe that, if $R=0$, then (A4) in fact implies $\beta_{i}(t) \leq 1$ for all $i \in N$ and $t \in M$, since $c_{i} \leq E$ for all $i$.

Proof of Theorem 4.5. Necessity follows from Lemmas 3.1 and 4.1-4.4. For sufficiency, it is convenient to consider the following tables associated with a claims profile satisfying (A1)-(A4).

\begin{tabular}{cccc}
\hline Case & $\beta_{N}(t)$ & $\beta_{i}(t)$ & marginal loss \\
\hline (i) & $K+1$ & 1 & $\frac{1}{K+1}$ \\
(ii) & $K$ & 1 & $\frac{1}{K}$ \\
(iii) & $K$ & 2 & $\frac{2}{K}-\frac{1}{K-1}$ \\
\hline
\end{tabular}

and

\begin{tabular}{cccc}
\hline Case & $\beta_{N}\left(t^{\prime}\right)$ & $\beta_{i}\left(t^{\prime}\right)$ & marginal gain \\
\hline (a) & $K$ & 0 & $\frac{1}{K+1}$ \\
(b) & $K$ & 1 & $\frac{2}{K+1}-\frac{1}{K}$ \\
(c) & $K$ & 2 & $\frac{3}{K+1}-\frac{2}{K}$ \\
(d) & $K+1$ & 0 & $\frac{1}{K+2}$ \\
(e) & $K+1$ & 1 & $\frac{2}{K+2}-\frac{1}{K+1}$ \\
\hline
\end{tabular}

The upper table gives the marginal losses incurred by removing a claim in any of the three possible cases (i)-(iii). The lower table gives the marginal gains by adding a claim in each of the five possible cases (a)-(e). It is straightforward to check that condition (2) is satisfied for each shift of a claim from one of the cases (i)-(iii) to one of the cases (a)-(e).

Theorem 4.5 gives a complete characterization of the Nash equilibrium claims profiles for restricted claims problems. A description of the associated payoff vectors is as follows. Let $r_{i}$ denote the part of player $i$ 's total claim $c_{i}$ invested in intervals with number of claims $K+1$. Obviously, $r_{i} \leq c_{i}$ and by (A2) in 
Theorem 4.5, $r_{i} \leq R$. Hence, $r_{i} \leq \min \left\{c_{i}, R\right\}$. On the other hand, by (A3), we have $c_{i}-r_{i} \leq 2(E-R)$ : the part invested in intervals with number of claims equal to $K$ cannot exceed two times the remaining part $E-R$ of the estate. Hence, $r_{i} \geq \max \left\{c_{i}-2(E-R), 0\right\}$. Additionally, the $r_{i}$ should sum up to $(K+1) R$. Summarizing, each NEP corresponds to a vector $\left(r_{1}, \ldots, r_{n}\right)$ satisfying

$\sum_{i \in N} r_{i}=(K+1) R$, and $\max \left\{c_{i}-2(E-R), 0\right\} \leq r_{i} \leq \min \left\{c_{i}, R\right\}$ for all $i \in N$.

Conversely, each such vector gives rise to an NEP. Although there are many NEP associated with the same $\left(r_{1}, \ldots, r_{n}\right)$ since there is quite some freedom in choosing the intervals and distributing the claims, all these NEP have the same payoffs, namely

$$
x_{i}=\frac{r_{i}}{K+1}+\frac{c_{i}-r_{i}}{K} .
$$

Equations (3) and (4) imply that the set of payoff vectors attainable by an NEP is determined by linear inequalities and, in particular, is a polytope. These equations are also convenient to check if a certain payoff, e.g. the payoff prescribed by some well known rule, can be obtained in an equilibrium. The following example shows this for the proportional rule: in particular, it implies that the proportional rule does not have to result in an equilibrium payoff vector.

Example 4.6 Consider the restricted claims problem $(E, c)$ with $E=6$ and $c=(1,2,5)$. The so-called proportional rule distributes the estate proportional to the claims and assigns the payoff vector $x=\left(\frac{6}{8}, \frac{12}{8}, \frac{30}{8}\right)$. For this problem, $K=1$ and $R=2$. Hence, $0 \leq r_{1} \leq 1,0 \leq r_{2} \leq 2$, and $0 \leq r_{3} \leq 2$, while $r_{1}+r_{2}+r_{3}=4$. Hence, in an NEP player 1's payoff is in $\left[\frac{1}{2}, 1\right]$, player 2's payoff is in $\left[1, \frac{3}{2}\right]$, and player 3's payoff is in $\left[4, \frac{9}{2}\right]$. Since $\frac{30}{8}<4$, it follows that the proportional payoff vector is not an equilibrium payoff vector.

Remark 4.7 If there are two players $(n=2)$ then it is easy to see by direct inspection or by using the results above that the only choice for $\left(r_{1}, r_{2}\right)$ is: $r_{1}=r_{2}=R$. This results in the unique equilibrium payoffs $x_{i}=\left(E+c_{i}-c_{j}\right) / 2$ for $i=1,2, j \neq i$. These payoffs coincide with the payoffs of the so-called minimal overlap rule. See Alcalde et al. (2008) for a recent characterization of this rule.

We conclude this section with an example which indicates that NEP in which some players have double claims on part of the estate tend to favor 'big' players.

Example 4.8 Consider the claims problem $(E, c)$ with $E=1, n=17, c_{1}=$ $c_{2}=0.9$, and $c_{3}=\ldots=c_{17}=0.2$. Then $K=4$ and $R=0.8$. From the point of view of players 1 and 2, the payoff-best NEP is one where both players put 2 claims on part $E-R=0.2$ of the estate. Consider for instance the NEP $(y, \beta, 7)$ with $y=(0,0.2,0.4,0.5,0.6,0.7,0.8,1), \beta_{1}=\beta_{2}=(1,1,1,0,0,0,2), \beta_{3}=\ldots=$ $\beta_{5}=(1,0,0,0,0,0,0), \beta_{6}=\ldots=\beta_{8}=(0,1,0,0,0,0,0), \beta_{9}=\ldots=\beta_{11}=$ 
$(0,0,1,1,0,0,0), \beta_{12}=\ldots=\beta_{14}=(0,0,0,0,1,1,0), \beta_{15}=(0,0,0,1,1,0,0)$, $\beta_{16}=(0,0,0,0,1,1,0)$, and $\beta_{17}=(0,0,0,1,0,1,0)$. Then players 1 and 2 have payoffs 0.2 each, and the other players receive 0.04 each. In an NEP where players 1 and 2 do not have double claims, they receive at most $\frac{1}{4} \cdot 0.2+\frac{1}{5} \cdot 0.7=$ 0.19 ; in such an equilibrium the other players receive at most $\frac{1}{4} \cdot 0.2=0.05$.

\section{General claims problems}

For the general claims problem, where claims are not restricted (not all $c_{i}$ smaller than or equal to $E$ ), a first observation to make is that NEP may fail to exist.

Example 5.1 Consider the two-player claims problem $(E, c)$ with $E=1$ and $c=\left(1.1, \frac{1}{2}\right)$. Assume (w.l.o.g.) that player 2 puts claims on the interval $[0, d]$ with $d \leq \frac{1}{2}$. Obviously, to obtain an NEP, player 1 should claim $[d, 1]$ exactly once. Suppose there were a part of $[0, d]$ claimed more than once by player 2 , say (w.l.o.g.) $[0, e]$ with $e \leq d$. Player 2's marginal loss on $[0, e]$ is at most $\frac{1}{6}$, attained if player 1 has one claim on $[0, e]$ and player 2 has two claims on $[0, e]$ : then 2's marginal loss is $\frac{2}{3}-\frac{1}{2}$. His marginal gain by adding a claim on $[d, 1]$ would be $\frac{1}{2}$, hence this would not be part of an NEP. Therefore, we have $d=\frac{1}{2}$, and player 2 claims $\left[0, \frac{1}{2}\right]$ exactly once. Next, we may assume (w.l.o.g.) that player 1 claims $[0,0.1]$ twice and $[0.1,1]$ once: this is, essentially, the unique best reply. But then, player 2's marginal loss by removing a claim from $[0,0.1]$ would be $\frac{1}{3}$, while his marginal gain by adding a claim on $\left[\frac{1}{2}, 1\right]$ would be $\frac{1}{2}$. Hence, there is no NEP.

Recall from Lemma 3.2 that a claims profile $(y, \beta, m)$ for a claims problem $(E, c)$ satisfies $\beta_{\max }-\beta_{\min } \leq 2$, hence $\beta_{\max }-\beta_{\min } \in\{0,1,2\}$. These three cases are considered in the following subsection. ${ }^{4}$

\subsection{A complete characterization of NEP}

\subsubsection{The case $\beta_{\max }-\beta_{\min }=2$}

We present a characterization of all NEP satisfying $\beta_{\max }-\beta_{\min }=2$.

Proposition 5.2 Let $(y, \beta, m)$ be an NEP for the claims problem $(E, c)$ such that $\beta_{\max }-\beta_{\min }=2$. Then there are players $i, j \in N, i \neq j$, such that the following three conditions hold.

(i) For each $t \in M$ with $\beta_{N}(s)=\beta_{\max }, \beta_{i}(s)=\beta_{j}(s)=\frac{\beta_{\max }}{2}$.

(ii) For each $s \in M$ with $\beta_{N}(s)=\beta_{\min }, \beta_{i}(s)=\beta_{j}(s)=\frac{\beta_{\min }}{2}$.

(iii) For each $s \in M$ with $\beta_{N}(s)=\beta_{\min }+1$,

$$
\beta_{i}(s), \beta_{j}(s) \in\left\{\frac{\beta_{\min }}{2}, \frac{\beta_{\max }}{2}\right\} .
$$

\footnotetext{
${ }^{4}$ It can be shown that also in the general case the payoffs associated with NEP form a (possible empty) polytope. See Atlamaz (2004).
} 
Note that this proposition implies that for every player $k \neq i, j, \beta_{k}(s)=0$ if $\beta_{N}(s) \in\left\{\beta_{\min }, \beta_{\max }\right\}$, and $\beta_{k}(s) \in\{0,1\}$ if $\beta_{N}(s)=\beta_{\min }+1\left(=\beta_{\max }-1\right)$. Hence, there are two 'big players' $i$ and $j$, and all other players have at most one claim per interval, and zero claims in all minimally or maximally claimed intervals.

Proof of Proposition 5.2. Let $t, t^{\prime} \in M$ with $\beta_{N}(t)=\beta_{\max }$ and $\beta_{N}\left(t^{\prime}\right)=\beta_{\min }$.

(a) First note that there cannot be any player $i \in N$ with $\beta_{i}(t)-\beta_{i}\left(t^{\prime}\right) \geq 2$ : this would lead to a contradiction in exactly the same way as in part (ii) of the proof of Lemma 3.2.

(b) Hence, by Lemma 3.3, there must be $i, j \in N, i \neq j$, with $\beta_{i}(t)=$ $\beta_{i}\left(t^{\prime}\right)+1$ and $\beta_{j}(t)=\beta_{j}\left(t^{\prime}\right)+1$. By $(* *)$ in the proof of Lemma 3.2, we have

$$
\beta_{i}\left(t^{\prime}\right), \beta_{j}\left(t^{\prime}\right) \geq \beta_{\min } \frac{\beta_{\max }\left(\beta_{\max }-1\right)-\left(\beta_{\min }+1\right)\left(\beta_{\max }-1\right)}{\beta_{\max }\left(\beta_{\max }-1\right)-\beta_{\min }\left(\beta_{\min }+1\right)} .
$$

Since the right hand side of this inequality is equal to $\frac{\beta_{\min }}{2}$, we must have $\beta_{i}\left(t^{\prime}\right)=\beta_{j}\left(t^{\prime}\right)=\frac{\beta_{\min }}{2}$, and thus $\beta_{i}(t)=\beta_{j}(t)=\frac{\beta_{\max }}{2}$. For any other $s \in M$ with $\beta_{N}(s)=\beta_{\max }$ it follows that $\beta_{i}(s)=\beta_{j}(s)=\frac{\beta_{\max }}{2}$ by applying (a) and Lemma 3.3 to $s$ and $t^{\prime}$. Similarly, by the same arguments it follows that for any other $s \in M$ with $\beta_{N}(s)=\beta_{\min }$ we have $\beta_{i}(s)=\beta_{j}(s)=\frac{\beta_{\min }}{2}$. This proves (i) and (ii).

(c) Let $t^{\prime \prime} \in M$ with $\beta_{N}\left(t^{\prime \prime}\right)=\beta_{\min }+1\left(=\beta_{\max }-1\right)$. Suppose $\beta_{i}\left(t^{\prime \prime}\right)<\frac{\beta_{\min }}{2}$. Then $\beta_{i}\left(t^{\prime \prime}\right)+1<\beta_{\max }$ and thus

$$
\frac{\frac{\beta_{\max }}{2}-\left(\beta_{i}\left(t^{\prime \prime}\right)+1\right)}{\beta_{\max }}<\frac{\frac{\beta_{\max }}{2}-\left(\beta_{i}\left(t^{\prime \prime}\right)+1\right)}{\beta_{\max }-1}
$$

which implies

$$
\frac{\beta_{i}(t)}{\beta_{N}(t)}-\frac{\beta_{i}(t)-1}{\beta_{N}(t)-1}<\frac{\beta_{i}\left(t^{\prime \prime}\right)+1}{\beta_{\min }+2}-\frac{\beta_{i}\left(t^{\prime \prime}\right)}{\beta_{\min }+1}
$$

violating (2). Similarly, if $\beta_{i}\left(t^{\prime \prime}\right)>\frac{\beta_{\max }}{2}$ then (2) is violated for $t^{\prime \prime}$ (in the role of $t$ in $(2))$ and $t^{\prime}$. We conclude that $\beta_{i}\left(t^{\prime \prime}\right) \in\left\{\frac{\beta_{\min }}{2}, \frac{\beta_{\max }}{2}\right\}$ and, similarly, $\beta_{j}\left(t^{\prime \prime}\right) \in\left\{\frac{\beta_{\min }}{2}, \frac{\beta_{\max }}{2}\right\}$.

It turns out that the three conditions in Proposition 5.2 are also sufficient for an NEP.

Proposition 5.3 Let $(y, \beta, m)$ be a claims profile for claims problem $(E, c)$ and assume that the following conditions are satisfied:

(a) $\beta_{\text {max }}-\beta_{\text {min }}=2$.

(b) There are $i, j \in N$ such that:

(i) For each $s \in M$ with $\beta_{N}(s)=\beta_{\max }, \beta_{i}(s)=\beta_{j}(s)=\frac{\beta_{\max }}{2}$. 
(ii) For each $s \in M$ with $\beta_{N}(s)=\beta_{\text {min }}, \beta_{i}(s)=\beta_{j}(s)=\frac{\beta_{\min }}{2}$.

(iii) For each $s \in M$ with $\beta_{N}(s)=\beta_{\text {min }}+1$,

$$
\beta_{i}(s), \beta_{j}(s) \in\left\{\frac{\beta_{\min }}{2}, \frac{\beta_{\max }}{2}\right\} .
$$

Then $(y, \beta, m)$ is an NEP.

Proof. It is straightforward to check that (2) holds in all possible cases.

An NEP $(y, \beta, m)$ looks (w.l.o.g.) as follows. Take $m=3$ and $0=y_{0}<y_{1}<$ $y_{2}<y_{3}=E$; let $L \in \mathbb{N}, \beta_{N}(1)=2 L+2, \beta_{N}(2)=2 L+1$, and $\beta_{N}(3)=2 L$. If $i$ and $j$ are the big players, then there must be $0 \leq \alpha_{h} \leq 1$ for each $h \in N$ with $\sum_{h \in N} \alpha_{h}=1$ such that $c_{i}=L E+y_{1}+\alpha_{i}\left(y_{2}-y_{1}\right), c_{j}=L E+y_{1}+$ $\alpha_{j}\left(y_{2}-y_{1}\right)$, and $c_{h}=\alpha_{h}\left(y_{2}-y_{1}\right)$ for all $h \neq i, j$. This implies that there are $0<d_{i}, d_{j}<E$ with $c_{i}=L E+d_{i}, c_{j}=L E+d_{j}$, and $\sum_{h \neq i, j} c_{h}+\max \left\{d_{i}, d_{j}\right\}<$ $E$. Conversely, if all these conditions are satisfied, then we can construct an NEP by taking $y_{1}=\min \left\{d_{i}, d_{j}\right\}$ and $y_{2}=\sum_{h \neq i, j} c_{h}+\max \left\{d_{i}, d_{j}\right\}$. Hence, we have the following corollary, which characterizes all claims problems that have an NEP as in Proposition 5.3.

Corollary 5.4 Claims problem $(E, c)$ has an NEP $(y, \beta, m)$ with $\beta_{\max }-\beta_{\min }=2$ if and only if there are $i, j \in N, L \in \mathbb{N}$, and $d_{i}, d_{j} \in \mathbb{R}$ with $0<d_{i}, d_{j}<E$, such that (i) $c_{i}=L E+d_{i}$ and $c_{j}=L E+d_{j}$, and (ii) $\sum_{h \in N \backslash\{i, j\}} c_{h}+\max \left\{d_{i}, d_{j}\right\}<E$.

\subsubsection{The case $\beta_{\max }-\beta_{\min }=0$}

This is a very special case since all parts of the estate are claimed equally. Hence, the total claim $\sum_{i \in N} c_{i}$ must be an integer multiple of $E$. We will present our characterization without proof. ${ }^{5}$

Proposition 5.5 Let $(y, \beta, m)$ be a claims profile for the claims problem $(E, c)$ such that $\beta_{\max }-\beta_{\min }=0$. Then $(y, \beta, m)$ is an NEP if and only if the following two conditions are satisfied.

(i) $\beta_{i}(t)-\beta_{i}\left(t^{\prime}\right) \leq 1$ for all $i \in N$ and all $t, t^{\prime} \in M$.

(ii) If $\beta_{i}(t)-\beta_{i}\left(t^{\prime}\right)=1$ for some $i \in N$ and $t, t^{\prime} \in M$, then

$$
\beta_{i}(t) \leq \frac{\beta_{\max }+1}{2}
$$

\subsubsection{The case $\beta_{\max }-\beta_{\min }=1$}

Also for this case we present the complete characterization without proof, see footnote 5 .

\footnotetext{
5 The proof follows familiar lines, always using (2), and is available upon request.
} 
Proposition 5.6 Let $(y, \beta, m)$ be a claims profile for the claims problem $(E, c)$ such that $\beta_{\max }-\beta_{\min }=1$. Then $(y, \beta, m)$ is an NEP if and only if the following seven conditions are satisfied.

(i) $\beta_{i}(t)-\beta_{i}\left(t^{\prime}\right) \leq 2$ for all $i \in N$ and all $t, t^{\prime} \in M$.

(ii) If $i \in N$ and $t, t^{\prime} \in M$ such that $\beta_{i}(t)=\beta_{i}\left(t^{\prime}\right)$ and $\beta_{N}(t)=\beta_{\text {min }}, \beta_{N}\left(t^{\prime}\right)=$ $\beta_{\max }$, then

$$
\beta_{i}(t) \leq \frac{3 \beta_{\min }^{2}+3 \beta_{\min }}{4 \beta_{\min }+2} .
$$

(iii) If $i \in N$ and $t, t^{\prime} \in M$ such that $\beta_{i}(t)=\beta_{i}\left(t^{\prime}\right)+1$ and $\beta_{N}(t)=\beta_{N}\left(t^{\prime}\right)=$ $\beta_{\text {max }}$, then

$$
\beta_{i}(t) \leq \frac{\beta_{\max }+1}{2} .
$$

(iv) If $i \in N$ and $t, t^{\prime} \in M$ such that $\beta_{i}(t)=\beta_{i}\left(t^{\prime}\right)+1$ and $\beta_{N}(t)=\beta_{\min }$, $\beta_{N}\left(t^{\prime}\right)=\beta_{\max }$, then

$$
\beta_{i}(t) \leq \frac{\beta_{\max }^{2}-1}{2 \beta_{\max }-1} .
$$

(v) If $i \in N$ and $t, t^{\prime} \in M$ such that $\beta_{i}(t)=\beta_{i}\left(t^{\prime}\right)+1$ and $\beta_{N}(t)=\beta_{N}\left(t^{\prime}\right)=$ $\beta_{\text {min }}$, then

$$
\beta_{i}(t) \leq \frac{\beta_{\max }}{2} .
$$

(vi) If $i \in N$ and $t, t^{\prime} \in M$ such that $\beta_{i}(t)=\beta_{i}\left(t^{\prime}\right)+2$, then $\beta_{N}(t)=\beta_{\text {min }}$, $\beta_{N}\left(t^{\prime}\right)=\beta_{\max }$, and

$$
\beta_{i}(t) \leq \frac{\beta_{\max }^{2}+3 \beta_{\max }-4}{4 \beta_{\max }-2} .
$$

In the next subsection we consider a special class of claims problems where it is easy to describe an explicit NEP.

\subsection{Claims containing an equal multiple of the estate}

Here we study a class of claims problems where existence of an NEP is guaranteed, and where an NEP can be easily described. These are the problems where each player's claim contains an equal multiple of the whole estate.

Proposition 5.7 Let $L \in \mathbb{N}$ and let $(E, c)$ be a claims problem with $c_{i}=L E+$ $d_{i}, 0<d_{i} \leq E$ for each $i \in N$. Let $\left(y, \beta^{\prime}, m\right)$ be an $N E P$ for the restricted claims problem $(E, d)$, and let $(y, \beta, m)$ be the claims profile for $(E, c)$ with $\beta_{i}(t)=L+\beta_{i}^{\prime}(t)$ for each $i \in N$ and $t \in M$. Then $(y, \beta, m)$ is an NEP for $(E, c)$. 
Proof. ${ }^{6}$ Observe that Theorem 4.5 applies to the NEP $\left(y, \beta^{\prime}, m\right)$ for $(E, d)$. This gives rise to the following marginal loss and gain tables for the claims profile $(y, \beta, m)$, where - as in Theorem $4.5-K$ is the total claim on minimally claimed intervals in $\left(y, \beta^{\prime}, m\right)$ and $K+1$ the total claim on maximally claimed intervals. The table of marginal losses is as follows.

\begin{tabular}{cccc}
\hline Case & $\beta_{N}(t)$ & $\beta_{i}(t)$ & marginal loss \\
\hline (i) & $n L+K+1$ & $L+1$ & $\frac{L+1}{n L+K+1}-\frac{L}{n L+K}$ \\
(ii) & $n L+K$ & $L+1$ & $\frac{L+1}{n L+K}-\frac{L}{n L+K-1}$ \\
(iii) & $n L+K$ & $L+2$ & $\frac{L+2}{n L+K}-\frac{L+1}{n L+K-1}$ \\
(iv) & $n L+K$ & $L$ & $\frac{L}{n L+K}-\frac{L-1}{n L+K-1}$ \\
(v) & $n L+K+1$ & $L$ & $\frac{L}{n L+K+1}-\frac{L-1}{n L+K}$ \\
\hline
\end{tabular}

The table shows all cases possible in view of the characterization in Theorem 4.5. Similarly, there are five cases to consider with respect to marginal gains:

\begin{tabular}{cccc}
\hline Case & $\beta_{N}\left(t^{\prime}\right)$ & $\beta_{i}\left(t^{\prime}\right)$ & marginal gain \\
\hline (a) & $n L+K$ & $L$ & $\frac{L+1}{n L+K+1}-\frac{L}{n L+K}$ \\
(b) & $n L+K$ & $L+1$ & $\frac{L+2}{n L+K+1}-\frac{L+1}{n L+K}$ \\
(c) & $n L+K$ & $L+2$ & $\frac{L+3}{n L+K+1}-\frac{L+2}{n L+K}$ \\
(d) & $n L+K+1$ & $L$ & $\frac{L+1}{n L+K+2}-\frac{L}{n L+K+1}$ \\
(e) & $n L+K+1$ & $L+1$ & $\frac{L+2}{n L+K+2}-\frac{L+1}{n L+K+1}$ \\
\hline
\end{tabular}

In order to check that for all shifts of a claim from one of the cases (i)-(v) to one of the cases (a)-(e), note that shifts from (iii) to (a) and from (iv) to (c) do not have to be considered since, by (A4) in Theorem 4.5, theses cases cannot occur simultaneously. As can be verified, the marginal losses are ordered according to (iii) $<$ (i) $<$ (ii) $<$ (v) $<$ (iv) and the marginal gains according to (a) $>$ (d) $>$ (b) $>$ (e) $>$ (c). It is, thus, sufficient to check that, first, the marginal loss in case (i) is at least as big as the marginal gain in case (a), and that, second, the marginal loss in case (iii) is at least as big as the marginal gain in case (d). The loss in (i) is actually equal to the gain in (a). By using the fact that $K \geq 4$ and $n \geq 5$, as follows from (A4), it can be shown that the marginal loss in (iii) is at least as big as the marginal gain in (d).

In an NEP in Proposition 5.7, each player claims $L$ times the whole estate. The remainders of the claims are distributed over $[0, E]$ such as to form an equilibrium of the kind in Theorem 4.5.

\footnotetext{
${ }^{6}$ An alternative proof can be given by applying the results - especially Propositions 5.5 and 5.6 - of the preceding section.
} 
Observe that for $n>2$ the classes of claims problems of Corollary 5.4 on the one hand and Proposition 5.7 on the other hand, are clearly disjoint: in the former there are exactly two 'big' players, whereas in the latter all players are 'big'. For $n=2$ there is overlap, resulting in different types of equilibria, as the following example shows.

Example 5.8 Let $n=2, E=1, c=(1.4,1.8)$. An equilibrium $(y, \beta, m)$ according to Corollary 5.4 (or Proposition 5.2) is as follows: $m=3 ; y=(0,0.4,0.8,1)$; $\beta_{1}=(2,1,1), \beta_{2}=(2,2,1)$. In this NEP, $\beta_{\max }=4$ and $\beta_{\min }=2$.

An equilibrium $(y, \beta, m)$ according to Proposition 5.7 is as follows: $m=3$; $y_{0}=(0,0.2,0.4,1) ; \beta_{1}=(2,2,1), \beta_{2}=(2,1,2)$. In this NEP, $\beta_{\max }=4$ and $\beta_{\min }=3$.

The next example shows that also if $n>2$ there may be equilibria different from the ones described in Proposition 5.7.

Example 5.9 Let $n=4, E=4, c=(5.1,5.1,5.1,5.8)$. An equilibrium of the type of Proposition 5.7 is $(y, \beta, m)$ with $m=4, y=(0,1.1,2.2,3.3,4)$, $\beta_{1}=(2,1,1,1), \beta_{2}=(1,2,1,1), \beta_{3}=(1,1,2,1)$, and $\beta_{4}=(2,1,1,2)$.

Another equilibrium is $(y, \beta, m)$ with $m=2, y=(0,2.9,4), \beta_{1}=\beta_{2}=\beta_{3}=$ $(1,2)$, and $\beta_{4}=(2,0)$. In this NEP, player 4 puts no claims on the interval $[2.9,4]$.

There are claims problems, other than those described in Proposition 5.7, where NEP with $\beta_{\max }-\beta_{\min }=1$ exist. An example is the following.

Example 5.10 Let $n=3, E=1, c=(2.6,3.7,4.2)$. The total claim is 10.5. Clearly, by Lemma 3.2 and Corollary 5.4 , if there is an NEP $(y, \beta, m)$, it must be the case that $\beta_{\max }-\beta_{\min }=1$. An example of such an NEP is as follows: $m=4$, $y=(0,0.3,0.5,0.6,1), \beta_{1}=(3,3,3,2), \beta_{2}=(4,3,3,4)$, and $\beta_{3}=(4,5,4,4)$. This can be checked directly, or by applying Proposition 5.6.

\subsection{An asymptotic result}

We conclude this section by showing that - not unexpectedly - the distributions resulting from NEPs converge to the proportional distribution if the claims tend to infinity.

Theorem 5.11 Let $(E, c),\left(E, c^{1}\right),\left(E, c^{2}\right), \ldots$ be a sequence of claims problems with $\lim _{k \rightarrow \infty} c_{i}^{k}=\infty$ for all $i \in N$ and $\lim _{k \rightarrow \infty} c^{k} / \sum_{j \in N} c_{j}^{k}=c$. For each $k \in \mathbb{N}$ let $\left(y^{k}, b^{k}, m^{k}\right)$ be an $N E P$ for $\left(E, c^{k}\right)$ with payoff vector $x^{k}$. Then $\lim _{k \rightarrow \infty} x^{k}=E c$.

Proof. By Propositions 5.2, 5.5 and 5.6, for all $t, t^{\prime} \in M$ and all $i \in N$, we have $\left|\beta_{i}^{k}(t)-\beta_{i}^{k}\left(t^{\prime}\right)\right| \leq 2$ for all $k$. It follows, in particular, that $x_{i}^{k} \rightarrow E c_{i} / \sum_{j \in N} c_{j}$ for every $i \in N$ as $k \rightarrow \infty$. 


\section{Some variations}

In the model considered so far, in a claims problem $(E, c)$ a player $i$ can put any nonnegative integer claim on some part of the estate, as long as total claims do not exceed $c_{i}$. We can express this by saying that the claim height is equal to 1 and players put multiples of this claim height on parts of the estate. A natural question to ask is what would happen if we change this claim height to some number $0<\gamma \leq 1$. We can go a step further and assume that players can choose from a finite or infinite number of claim heights, or even vary claim heights over each point of the interval $[0, E]$ for an estate $E$. This section is devoted to a discussion of these variations.

\subsection{Fixed claim height smaller than 1}

Suppose the claim height is $\gamma$ with $0<\gamma \leq 1$. For a claims problem $(E, c)$ and a claims profile $(y, \beta, m), \beta_{i}(t) \in\{0\} \cup \mathbb{N}$ is now interpreted as the number of claims of height $\gamma$ put by player $i \in N$ on interval $t \in M$. In order to distinguish such a claim profile from a claim profile with claim height 1 we will write $(y, \beta, m ; \gamma)$. The associated payoff distribution is still given by (1). The feasibility constraint becomes $c_{i}=\sum_{t=1}^{m} \beta_{i}(t) \gamma\left(y_{t}-y_{t-1}\right)$ for each player $i$.

The obvious condition ${ }^{7}$ for an NEP similar to (2) is

$$
\frac{\beta_{i}(t) \gamma}{\beta_{N}(t) \gamma}-\frac{\beta_{i}(t) \gamma-\gamma}{\beta_{N}(t) \gamma-\gamma} \geq \frac{\beta_{i}\left(t^{\prime}\right) \gamma+\gamma}{\beta_{N}\left(t^{\prime}\right) \gamma+\gamma}-\frac{\beta_{i}\left(t^{\prime}\right) \gamma}{\beta_{N}\left(t^{\prime}\right) \gamma},
$$

but this inequality is of course equivalent to (2). Together with the feasibility condition this implies that $(y, \beta, m ; \gamma)$ is an NEP for $(E, c)$ if and only if $(y, \beta, m)=(y, \beta, m ; 1)$ is an NEP for $(E, c / \gamma)$. A consequence of these considerations and Theorem 5.11 is that equilibrium distributions converge to the proportional distribution as the claim height goes to zero. Formally, we have the following result.

Corollary 6.1 Let $1 \geq \gamma^{1}>\gamma^{2}>\ldots$ with $\lim _{k \rightarrow \infty} \gamma^{k}=0$ and let $\left(y^{k}, \beta^{k}, m^{k}\right.$; $\left.\gamma^{k}\right)$ be an $N E P$ for $(E, c)$ for each $k \in \mathbb{N}$, with payoff distribution $x^{k}$. Then $\lim _{k \rightarrow \infty} x^{k}=E c / \sum_{i \in N} c_{i}$.

\subsection{Arbitrary claim height}

For a claims problem $(E, c)$ and a claims profile $(y, \beta, m)$, we now assume that $\beta_{i}(t) \in \mathbb{R}_{+}=\{x \in \mathbb{R} \mid x \geq 0\}$ for all $i \in N$ and $t \in M$. We denote such a claims profile by $\left(y, \beta, m ; \mathbb{R}_{+}\right)$. The payoff distribution is given by (1). The feasibility constraint is still $c_{i}=\sum_{t=1}^{m} \beta_{i}(t)\left(y_{t}-y_{t-1}\right)$ for each player $i$. The condition 8 similar to (2) for an NEP is now

$$
\frac{\beta_{i}(t)}{\beta_{N}(t)}-\frac{\beta_{i}(t)-\varepsilon}{\beta_{N}(t)-\varepsilon} \geq \frac{\beta_{i}\left(t^{\prime}\right)+\varepsilon}{\beta_{N}\left(t^{\prime}\right)+\varepsilon}-\frac{\beta_{i}\left(t^{\prime}\right)}{\beta_{N}\left(t^{\prime}\right)}
$$

\footnotetext{
${ }^{7} \mathrm{Cf}$. footnote 2 .

${ }^{8} \mathrm{Cf}$. footnote 2 .
} 
for each $i \in N, t \in M$ with $\beta_{i}(t)>0, t^{\prime} \in M$, and $0<\varepsilon<\beta_{i}(t)$. This inequality can be rewritten to

$$
\frac{\beta_{N}(t)-\beta_{i}(t)}{\beta_{N}(t)\left(\beta_{N}(t)-\varepsilon\right)} \geq \frac{\beta_{N}\left(t^{\prime}\right)-\beta_{i}\left(t^{\prime}\right)}{\beta_{N}\left(t^{\prime}\right)\left(\beta_{N}\left(t^{\prime}\right)+\varepsilon\right)}
$$

and this is satisfied for each $\varepsilon>0$ if and only if

$$
\frac{\sum_{j \in N \backslash\{i\}} \beta_{j}(t)}{\left(\beta_{N}(t)\right)^{2}} \geq \frac{\sum_{j \in N \backslash\{i\}} \beta_{j}\left(t^{\prime}\right)}{\left(\beta_{N}\left(t^{\prime}\right)\right)^{2}} .
$$

Note that, if $\beta_{i}\left(t^{\prime}\right)>0$ as well, then also the converse of this inequality has to hold, hence we have equality.

Consider any claims profile $\left(y, \beta, m ; \mathbb{R}_{+}\right)$and a player $i \in N$. Given the claims profile restricted to $N \backslash\{i\}$, namely $\left(y,\left(\beta_{j}\right)_{j \in N \backslash\{i\}}, m ; \mathbb{R}_{+}\right)$, and assuming $^{9}$ that $\sum_{j \in N \backslash\{i\}} \beta_{j}(t)>0$ for all $t \in M$, player $i$ can guarantee a payoff of $\left(c_{i} / \sum_{j \in N} c_{j}\right) E$ by claiming according to $\beta_{i}^{\prime}$ defined by

$$
\beta_{i}^{\prime}(t)=\left(c_{i} / \sum_{j \in N \backslash\{i\}} c_{j}\right) \sum_{j \in N \backslash\{i\}} \beta_{j}(t)
$$

for all $t \in M$. This way, player $i$ obtains his proportional share of every interval, and thus of the estate $E$. Since this is true for every player $i$, every NEP $\left(y, \beta, m ; \mathbb{R}_{+}\right)$for $(E, c)$ must result in the proportional distribution. Moreover, it follows easily from (5) that $\left(y, \beta, 1 ; \mathbb{R}_{+}\right)$- thus, each player $i$ putting a claim of height $c_{i}$ on the whole interval $[0, E]-$ is an NEP. We call this profile the uniform claims profile. In fact, the uniform claims profile is the unique ${ }^{10} \mathrm{NEP}$ among the claims profiles $\left(y, \beta, m ; \mathbb{R}_{+}\right)$. This can be seen as follows. Let $\left(y, \beta, m ; \mathbb{R}_{+}\right)$ be an NEP different from the uniform claims profile. Clearly, we may assume $\beta_{N}(t)>0$ for all $t \in M$. Now there must be an $i \in N$ and $t, t^{\prime} \in M$ such that $\sum_{j \in N \backslash\{i\}} \beta_{j}(t)>\sum_{j \in N \backslash\{i\}} c_{j} / E>\sum_{j \in N \backslash\{i\}} \beta_{j}\left(t^{\prime}\right)$. (This is so since against a constant total claim of $N \backslash\{i\}$, player $i$ 's unique best reply is to claim uniformly - this follows from (5).) By claiming proportionally according to $\beta_{i}^{\prime}$ as above, player $i$ obtains his proportional payoff $\left(c_{i} / \sum_{j \in N} c_{j}\right) E$. In that case, we have

$$
\frac{\sum_{j \in N \backslash\{i\}} \beta_{j}(s)}{\left(\beta_{N}(s)\right)^{2}}=\frac{1}{\sum_{j \in N \backslash\{i\}} \beta_{j}(s)\left(1+c_{i} / \sum_{j \in N} c_{j}\right)^{2}}
$$

for $s=t, t^{\prime}$. Therefore, (5) is violated, implying that player $i$ can improve his payoff by transferring part of his claim from $t$ to $t^{\prime}$. This, in turn, implies that his equilibrium payoff is higher than proportional, a contradiction.

Summarizing, we have the following result.

\footnotetext{
${ }^{9}$ If this assumption is not satisfied then player $i$ can even do better by putting a claim of very small height on the non-claimed part(s) of the interval $[0, E]$.

${ }^{10} \mathrm{In}$ the sense of numbers of claims on each part of the estate.
} 
Proposition 6.2 Among all claims profiles $\left(y, \beta, m ; \mathbb{R}_{+}\right)$for claims problem $(E, c)$, the uniform claims profile, resulting in the proportional payoff distribution $E c / \sum_{i \in N} c_{i}$, is the unique NEP.

\subsection{Arbitrary claim height point-wise}

We now assume that a claims profile for $(E, c)$ is any $n$-tuple of continuous functions $f_{i}:[0, E] \rightarrow \mathbb{R}_{+}$such that for each $i \in N, \int_{0}^{E} f_{i}(t) d t=c_{i}$. Denote $f_{N}(t)=\sum_{i \in N} f_{i}(t)$ for each $t \in[0, E]$ and, for each $i \in N$ and $t \in[0, E]$,

$$
\hat{f}_{i}(t)=\left\{\begin{array}{cl}
\frac{f_{i}(t)}{f_{N}(t)} & \text { if } f_{i}(t)>0 \\
0 & \text { if } f_{i}(t)=0
\end{array}\right.
$$

Then the payoff distribution associated with $f=\left(f_{1}, \ldots, f_{n}\right)$ is given by

$$
x_{i}=\int_{0}^{E} \hat{f}_{i}(t) d t \text { for every } i \in N .
$$

Within this model the uniform claims profile is the profile $u=\left(u_{1}, \ldots, u_{n}\right)$ defined by $u_{i}(t)=c_{i} / E$ for all $i \in N$ and $t \in[0, E]$. In this case, by arguments analogous to those in the preceding subsection we obtain the following result.

Proposition 6.3 Among all claims profiles $f=\left(f_{1}, \ldots, f_{n}\right)$ for claims problem $(E, c)$, the uniform claims profile $u$, resulting in the proportional payoff distribution $E c / \sum_{i \in N} c_{i}$, is the unique $N E P$.

Remark 6.4 A variation in-between claim height 1 and arbitrary claim height is the following. For a claims problem $(E, c)$, player $i$ first divides the rectangle with height 1 and width $c_{i}$ into smaller rectangles by choosing a finite number of cuts on the horizontal interval $\left[0, c_{i}\right]$ and a finite number of cuts on the vertical interval $[0,1]$. Next, player 1 puts these rectangular claims on the estate $[0, E]$. It can be shown that still any NEP must result in the proportional distribution - the uniform claims profile may, however, not even be feasible. Among the variations discussed above, this is the minimal (coursest) variation that nevertheless results in proportional distribution in equilibrium. See Berden (2008) for the details.

\section{Concluding remarks}

Extending an approach initiated by O'Neill (1982), the present paper offers non-cooperative solutions to the claims (or bankruptcy) problem. An interesting additional observation is that many well known bankruptcy rules do not always pick equilibrium payoff distributions. Example 4.6 shows this for the proportional rule, but examples can be constructed for many other rules as well. See Proposition 7 in Atlamaz (2004), which shows this for the constrained equal losses rule and the truncated equal losses rule, the constrained equal 
awards rule and the truncated equal awards rule, and the Talmud rule, besides the proportional rule. ${ }^{11}$ This observation raises the question if there is an axiomatic, normative justification for the 'rule' found in this paper, namely the correspondence that assigns the set (polytope) of NEP payoff distributions to each claims problem.

In our approach the proportional division rule plays an important role, which is not surprising since it is used to define payoffs in the basic strategic game. This raises the issue of what happens if some other principle is used to define the payoffs in this game.

A third interesting issue arises if the implicit assumption of a homogeneous estate is dropped. In the present paper, players are indifferent as to the different parts of the interval $[0, E]$. Dropping this assumption leads to a model closely related to models of land division or, more generally, division of heterogeneous goods, see among others Berliant (1985), Berliant and Ten Raa (1988), Berliant et al. (1992), and Legut et al. (1994).

\section{References}

Alcalde, J., M. del Carmen Marco, and J.A. Silva (2008): "The Minimal Overlap Rule Revisited," Social Choice and Welfare, 31, 109-128.

Atlamaz, M. (2004): "Contests with Fixed Resources in Spatial Models," University of Rochester.

Aumann, R. and M. Maschler (1985): "Game Theoretic Analysis of a Bankruptcy Problem from the Talmud," Journal of Economic Theory, 36, 195-213.

Berden, C. (2008): Risk Aversion and Equilibria, Intertemporal Transfers, Strategic Claims. PhD-thesis, University of Maastricht. ISBN 978-909023485-4.

Berden, C., H. Peters, and D. Vermeulen (2008): "Non-Cooperative Claims Problems," mimeo, University of Maastricht.

Berliant, M. (1985): "An Equilibrium Existence Result for an Economy with Land," Journal of Mathematical Economics, 14, 53-56.

Berliant, M. and T. Ten Raa (1988): "A Foundation of Location Theory: Consumer Preferences and Demand," Journal of Economic Theory, 44, 336-353.

Berliant, M., W. Thomson and K. Dunz (1992): "On the Fair Division of a Heterogeneous Commodity," Journal of Mathematical Economics, 21, 201-206.

\footnotetext{
${ }^{11}$ One and the same example can be used to show this, e.g., $(E, c)=(1,(2.1,1.8,1.3))$.
} 
Borel, E. (1921): "La Théorie du Jeu et les Équations Intégrales à Noyau Symmétrique," Comptes Rendus de l'Académie des Sciences, 173, 13041308. English translation by L. Savage (1953): "The Theory of Play and Integral Equations with Skew Symmetric Kernels," Econometrica, 21, 97100.

Chun, Y. (1989): "A Non-cooperative Justification for Egalitarian Surplus Sharing," Mathematical Social Sciences, 17, 245-261.

Corchón, L. and C. Herrero (2004): "A Decent Proposal," Spanish Economic Review, 6, 107-125.

Cramton, P., R. Gibbons, and P. Klemperer (1987): "Dissolving a Partnership Efficiently," Econometrica, 55, 615-632.

Dagan, N., R. Serrano, O. Volij (1997): "A Non-cooperative View of Consistent Bankruptcy Rules," Games and Economic Behavior, 18, 55-72.

García-Jurado, I., J. González-Díaz, A. Villar (2006): "A Non-cooperative Approach to Bankruptcy Problems," Spanish Economic Review, 8, 189197.

Hotelling, H. (1929): "Stability in Competition," The Economic Journal, 39, $41-57$.

Legut, J., J.A.M. Potters, and S.H. Tijs (1994): "Economies with Land - A Game Theoretical Approach," Games and Economic Behavior, 6, 416430.

Merolla, J., M. Munger, and M. Tofias (2003): "Lotto, Blotto, or Frontrunner: An Analysis of Spending Patterns by the National Parties Committees in the 2000 Presidential Election," mimeo, Duke University.

O’Neill, B. (1982): "A Problem of Rights Arbitration from the Talmud," Mathematical Social Sciences, 2, 345-371.

Thomson, W. (2003): "Axiomatic and Game-theoretic Analysis of Bankruptcy and Taxation Problems: a survey," Mathematical Social Sciences, 45, 249297. 